\title{
STANDARDS OF DISTORTED SIGNAL FOR Calibration of Total Harmonic Distortion METERS
}

\author{
Pavel Horský \\ Central European Design Office \\ Videňská 127, CZ - 61900 BRNO \\ E-mail: horsky@cedo.cz
}

Abstract
In this article a survey and evaluation of some
progressive approaches to the standards of non-linear
distortion is reported. Some types of standards of
signals with the known total harmonic distortion used
for calibration of distortion measuring instruments are
proposed. This article is based on our previous work
directed at the development of a multifunctional THD
standard which is now used in the Czech Metrological
Institute.

\section{Introduction}

Non-linear distortion is a dimensionless quantity commonly used to characterize the spectral impurity of a signal

The measurement of the non-linear distortion is widely used, mainly in lf electronics and electroacoustics for characterization of different devices. The non-linear distortion is also significant in the metrology of If ac quantities (voltage, current, electric power, work and etc.).

Only little attention is paid to the calibration of distortion meters and sparse information on the topic is available. To our knowledge, no standard of non-linear distortion is available commercially at present.

\subsection{Definitions}

Total harmonic distortion is usually defined as the ratio of the rms of the harmonics (mostly only the first 5 are significant) to either the rms of the fundamental (THD') or the rms of the whole signal (THD). The ratio is usually converted to $\%$ or $\mathrm{dB}$. For small THD values, the difference between THD and THD' is negligible. The equations for THD and THD', respectively, read:

$$
T H D=\frac{\sqrt{\sum_{i=2}^{N} U_{i}^{2}}}{\sqrt{\sum_{i=1}^{N} U_{i}^{2}}} \quad T H D^{\prime}=\frac{\sqrt{\sum_{i=2}^{N} U_{i}^{2}}}{U_{1}}
$$

where $\quad U_{i}$ is the rms of the $i$-th harmonic.

Total harmonic distortion plus noise $(\mathrm{THD}+\mathrm{N})$ is usually defined as the ratio of the rms of the harmonics, hum and noise to the rms of the whole signal. In practice, distortion measurements are usually based on this parameter. The equation for $\mathrm{THD}+\mathrm{N}$ reads:

$$
(T H D+N)=\frac{\sqrt{\sum_{i=2}^{N} U_{i}^{2}+U_{n}^{2}+U_{s}^{2}}}{\sqrt{\sum_{i=1}^{N} U_{i}^{2}+U_{n}^{2}+U_{s}^{2}}}
$$

where $U_{i}$ is the rms of the $i$-th harmonic,

$U_{n}$ is the rms of noise and

$U_{s}$ is the rms of spurious and other disturbing signals.

Some other definitions, used to describe the nonlinearity of two-ports, have been reported [1] (e.g. SINAD or different intermodulation distortion definitions). They are, however, beyond the scope of this article.

\subsection{THD Measuring Instruments}

Many different methods of distortion measurement based on various distortion definitions are commonly used [1].

One possible approach is to measure the level of each harmonic with a spectral analyzer or a selective voltmeter. The THD' can then be calculated using Eq. (1b).

Another method consists in measuring distortion according to the THD $+\mathrm{N}$ definition given by $\mathrm{Eg}$. (2). In the first step, the whole rms signal level is measured and the measuring instrument sensitivity is set in such a way that the output reads $100 \%$. In the second step a notch filter suppresses the fundamental harmonic component of the signal and the rms level of such signal relative to the total signal level is measured. The measured value corresponds to the THD $+\mathrm{N}$. This procedure can be automated.

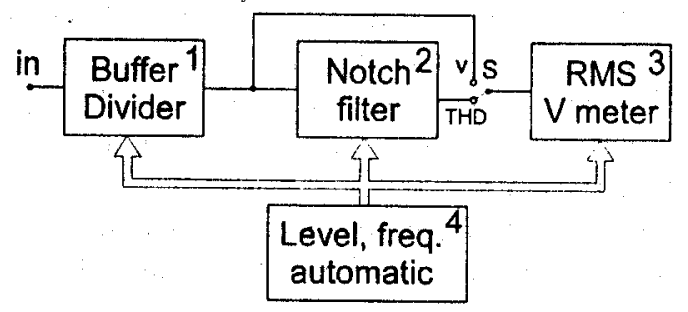

Fig. 1. THD+N meter main functional blocks 
Most of the commonly used THD+N measuring instruments operate in this way. A simplified block diagram of a THD $+\mathrm{N}$ measuring instrument is shown in Fig. 1.

\section{Calibration}

We need a standard of a distorted signal for the calibration of the tolal harmonic distortion meters. For the calibration using the method of functional blocks [3] we need a standard of the distorted signal containing the fundamental and one higher harmonic with a selectable frequency. We can obtain such a standard by summing two harmonic signals as shown below.

Calibration sources with more than two harmonic components are needed only in special cases. In practice, such standards can be used only in the acoustic frequency range in case the whole asserting calibrator frequency spectrum is situated in the specified frequency range of the calibrated measuring instrument.

A signal with known THD can be made in different ways, the following principles are preferred for metrology:

\section{3 "Pure" harmonic signal source}

\begin{tabular}{|c|c|c|c|c|}
\hline $\begin{array}{l}\text { RC } \\
\text { generator }\end{array}$ & $\begin{array}{l}\text { Filter } \\
\text { LP or } B P\end{array}$ & $\begin{array}{l}\text { - Pure } \\
\text { sinusoidal }\end{array}$ & $\begin{array}{l}\text { Har- } \\
\text { monic }\end{array}$ & $\begin{array}{l}\text { Cort. } \\
|\mathrm{dB}|\end{array}$ \\
\hline & & & 2 & 9.09 \\
\hline & & \multirow{3}{*}{$\begin{array}{l}\text { Wave analyzer } \\
\text { or selective } \\
\text { V-meter }\end{array}$} & 3 & 5.12 \\
\hline & & & 4 & 3.30 \\
\hline$R / 2$ & & & $\overline{5}$ & 2.29 \\
\hline \multicolumn{3}{|c|}{ Notch for $1^{\text {*1 }}$ harmonic } & 6 & 1.67 \\
\hline \multicolumn{3}{|c|}{ Measuring set for very low THD } & 7 & 1.27 \\
\hline
\end{tabular}

Fig. 2. Measurement of very low THD

For testing the lowest measurable distortion some types of commercially available RC generators with ultra low distortion can be used (possibly with additional lowor band-pass filters). $\mathrm{THD}+\mathrm{N}$ of the RC generator must be more than $10 \mathrm{~dB}$ lower than the smallest measurable THD of the calibrated distortion analyzer. The generator's THD can be measured with a selective microvoltmeter or a spectral analyzer. The dynamic range needs to be lowered by a notch filter with a passive double- $T$ network. Corrections for the signal attenuation in the notch filter (Fig. 2) have to be done for the calculation of THD from measured harmonic components. This method of THD measurement is applicable for measurement of ultra low distortions $\left(10^{-3} \%\right)$.

\section{Summation of two signals}

A distorted signal can be made by summing a pure harmonic signal (the fundamental harmonic component) and a signal representing the distortion. These signals can be generated by one or two signal sources. It is necessary to know the rms values of both signals or at least their ratio.

\subsection{Summation of two harmonic signals}

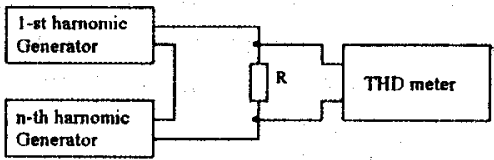

Fig. 3. Passive summation - generators are connected in series

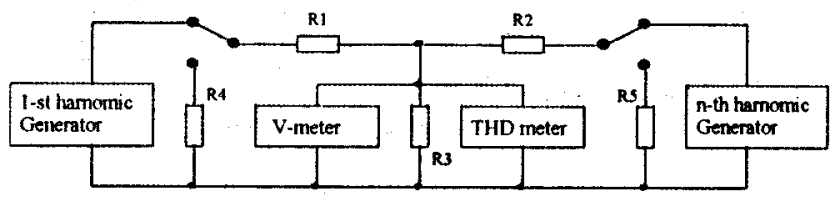

Fig. 4. Passive summation with $\mathrm{T}$ resistor network

This standard has two spectral components and can be used for calibration by the method of functional blocks [3]. Generally, it is not necessary to synchronize the two generators. If the generators can be used as both a source and a sink, it is possible to add the output signals in parallel (with a $\mathrm{T}$ resistor network) (Fig. 3) or in series (if one of them can work as floating) (Fig. 4). It is advantageous to use a pure harmonic signal generator with a constant amplitude (e.g. $1 \mathrm{~V}$ ) for the fundamental harmonic and an ac voltage calibrator for simulating the distortion component. The signals can be combined by a simple summation, which can be done by an active summator with ultra low distortion type of operational amplifier (Fig. 5). Such a network is advantageous as the generators do not influence each other and the output impedance of the summator is low. This "compound" distortion calibrator can be used in wide dynamic and frequency ranges (the summator must be tested separately). The achievable relative precision reaches from $0.01 \%$ to $2 \%$ (in the frequency range from $10 \mathrm{~Hz}$ to 100 $\mathrm{kHz}$ and for distortions greater than $0.01 \%$ ) [6].

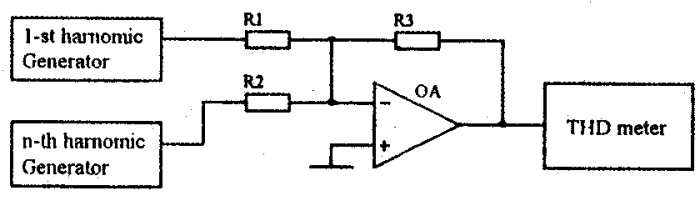

Fig. 5. Active summation - using summator with operational amplifier

\subsection{Summation of a harmonic and a distorted signal}

The distorted signal with a wide distortion range can also be made by summing (with a passive or an active summator) a pure harmonic signal and the same signal 
distorted in a non-linear circuit (Fig. 6). For non-linear distortion of the harmonic signal we use circuits with an output signal, for which the THD can be calculated exactly and the frequency spectrum of which is not too wide. It is useful to implement a half-way operational rectifier. To calculate the distortion of the output signal it is sufficient to know the summation ratio (determined just by the ratio of the summator resistors) of the harmonic and the distorted signal. This distortion standard principle can be used in a wide dynamic range and a wide range of THD. The output signal has wide frequency band with missing odd harmonics. Fig. 7 shows the frequency spectrum of the THD standard for $1 \%$ THD.

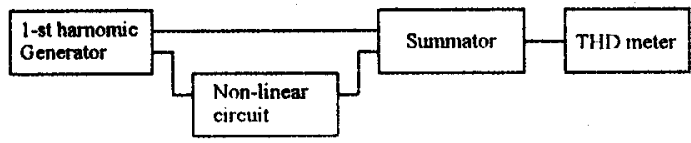

Fig. 6. Summation of a harmonic and a distorted signal

High-quality wide band operational amplifiers with low THD and Schottky-barrier diodes should be used. The inaccuracy of the output signal THD is determined by the operational rectifier error, summator error. the pure harmonic signal THD and the additional THD due to the circuitry. The achievable relative precision ranges from $0.1 \%$ to $2.5 \%$ (in the frequency range of $20 \mathrm{~Hz}$ to $20 \mathrm{kHz}$. and for the distortion of $0.03 \%$ to $10 \%|6|$.

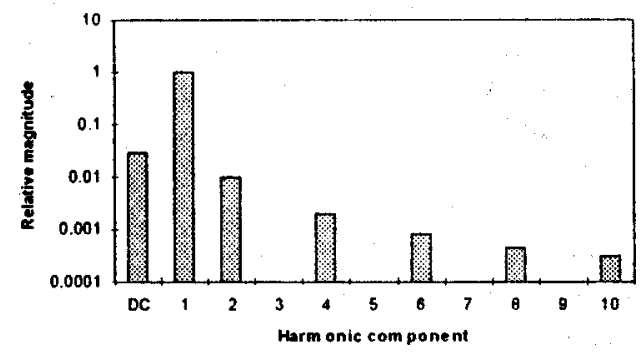

Fig. 7. Frequency spectrum of the THD standard with summing pure harmonic signal and half way rectified signal for $T H D=1 \%$

\section{Calculable standard of distorted signals}

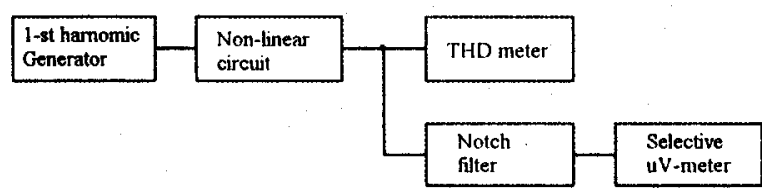

Fig. 8. Calculable THD standard block diagram

A signal source, the distortion of which can be determined without measuring the particular harmonics, has the character of an intrinsic standard. One example can be a calculable standard (Fig. 8) [2]. The calculable standard of a distorted signal can be made simply with a one-side limiter with selectable limiting level. For certain limiting levels $\left(V_{0} / V_{m}\right)$, some harmonics $(n-$ th) in spectra of the limited signal disappear and the corresponding values of THD can be exactly calculated as shown in Fig. 9. The left part of the graph illustrates the time course of the limited signal and the right part illustrates the dependence of the 5-th harmonic magnitude $\left(V_{5}\right.$, horizontal axis) on the limiting level.

\begin{tabular}{|c|r|r|r|r|r|r|}
\hline $\boldsymbol{n}$ & 4 & 5 & 5 & 5 & 6 & 6 \\
\hline $\boldsymbol{V}_{\boldsymbol{d}} \boldsymbol{V}_{m}$ & 0.408 & 0.612 & 0.000 & -0.612 & 0.727 & 0.266 \\
\hline $\mathrm{THD}[\%]$ & 22.861 & 14.539 & 39.908 & 68.206 & 9.9121 & 28.719 \\
\hline \hline$N$ & 6 & 6 & 7 & 7 & 7 & 7 \\
\hline$I^{\prime} \boldsymbol{V}_{m}$ & -0.266 & -0.727 & 0.798 & 0.443 & 0.000 & -0.443 \\
\hline $\mathrm{THD}[\%]$ & 51.761 & 74.513 & 7.1077 & 21.439 & 39.908 & 59.752 \\
\hline
\end{tabular}

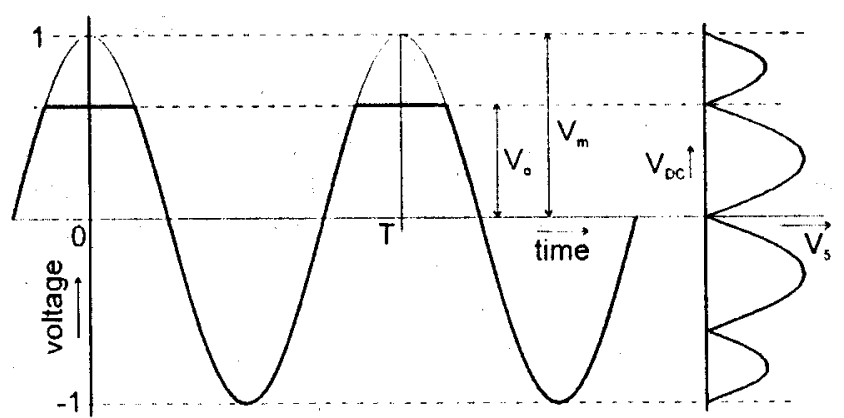

Fig. 9. One-side limited signal as a calculable standard of THD

A precise limiter can be realized simply with an operational rectifier with two inputs (the first one for the pure harmonic signal, the second one for the dc signal for establishing the limiling level). For this purpose it is necessary to use a precision wide-band operational amplifier (for the highest precision, an amplifier composed of monolithic and discrete components constructed as a voltage controlled current source should be used) and Schottky-barrier diodes. Sources of crrors are the same as in the operational rectificr alone. Another source of errors can be the inaccuracy of establishing the zero harmonic level [2]. This principle is usable in the frequency range up to $100 \mathrm{kHz}$, for larger THD (>1\%). Distorted signal has a wide frequency band. It is advantageous to utilize instruments, where zero level of the $n$-th harmonic can be found simply (spectral analyzers with THD calculation). The achievable relative precision ranges from $0.05 \%$ to $5 \%$ (in the frequency range of $20 \mathrm{~Hz}$ to $20 \mathrm{kHz}$ and for the distortion greater than $5 \%$ ) [6]

\section{Digital Methods}

The methods of generating an analog signal using digital to analog converters (DAC) are very progressive. The main advantage is that signals with almost arbitrary time course or frequency spectrum (up to one half of the sampling frequency) can be generated. The disadvantage is the circuit complexity, unwanted high harmonics and a difficult mathematical analysis of real DAC errors. 


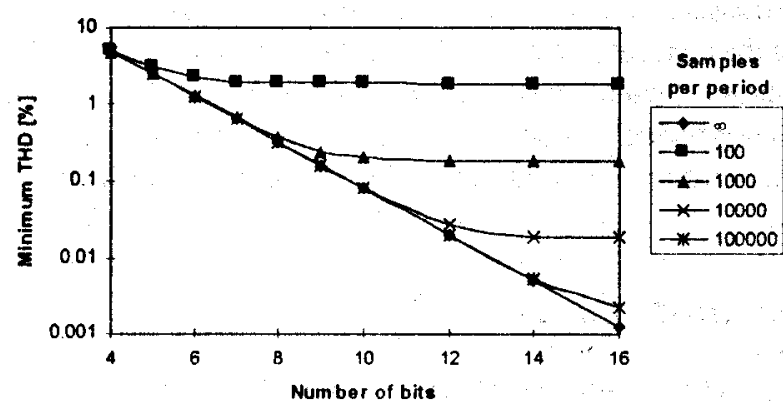

Fig. 10. Dependence of the minimum THD on the number of bits and the number of samples per period

Fig. 10 shows the theoretically calculated minimum value of THD on output of an ideal DAC (without low pass filter on the output) against the number of bits and the number of samples in one period of the output signal.

This method is useful in wide frequency and dynamic ranges, for higher values of distortion (greater than about $0.1 \%$ ) and for automated measurements. The achievable relative precision is dependent on the DAC used, e.g. for 12 bit. $40 \mathrm{MSa} / \mathrm{s}$ the relative precision is $1 \%-2.5 \%$ for distortion greater than $0.1 \%$ in the frequency range of $10 \mathrm{~Hz}$ to $100 \mathrm{kHz}$ [6].

\section{Realized multifunctional THD standard}

The aim of the multifunctional THD standard design is to develop a circuil allowing the realization of above mentioned analog THD standard principles. The main requirement is the highest possible accuracy of the output signal distortion. There are also some further requirements, e.g. additional filters.

Principle block diagram of the designed circuit is shown in Fig. 11. The inputs $\ln 1$ and $\ln 2$ are separated from the other parts of the circuit by buffers. In 1 . works as a universal input and an additional RC network is separating the relevant dc component. Quality low noise operational amplifiers (e.g. LT 1007) have to be used in the input buffers. The summator-input voltages can be measured by rms voltmeter on the output Monitor.

In order to achieve the highest possible precision the operational rectifier-limiter is composed of monolithic and discrele components, constructed as a voltagecontrolled current source [6]. In order to prevent loading of the rectifier output, there is a buffer with an operational amplifier on the operational rectifier output.

The summator is connected as a classical inverting summator with an operational amplifier. The operational amplifier $A D 797$ with ultra low distortion $(-120 \mathrm{~dB}$ on $20 \mathrm{kHz})$ and ultra low noise is chosen. An external $R$ decade can be connected to the second summator input.

In the calibrator function the output signal of the operational rectifier is summed by a passive resistor divider with the pure harmonic input signal.

The filter is designed for suppressing the line hum (frequency $50 \mathrm{~Hz}$ ) and all signals with frequencies higher than about $10 \mathrm{kHz}$. A passive double T network, a buffer with an operational amplifier with a high input resistance and two cascade-connected RC first-order lowpass networks are used.

The realized standard consists of the multifunctional THD standard (described above), a pure harmonic signal generator (in our case the ultra low distortion generator Krohn-Hite 4400A) and an optional dc or ac voltage calibrator, rms voltmeter and a $R$ decade.

The above mentioned THD standard can work as:

- A pure harmonic signal generator

- A calculable standard (discrete THD values)

- A standard with a one-side limited signal and

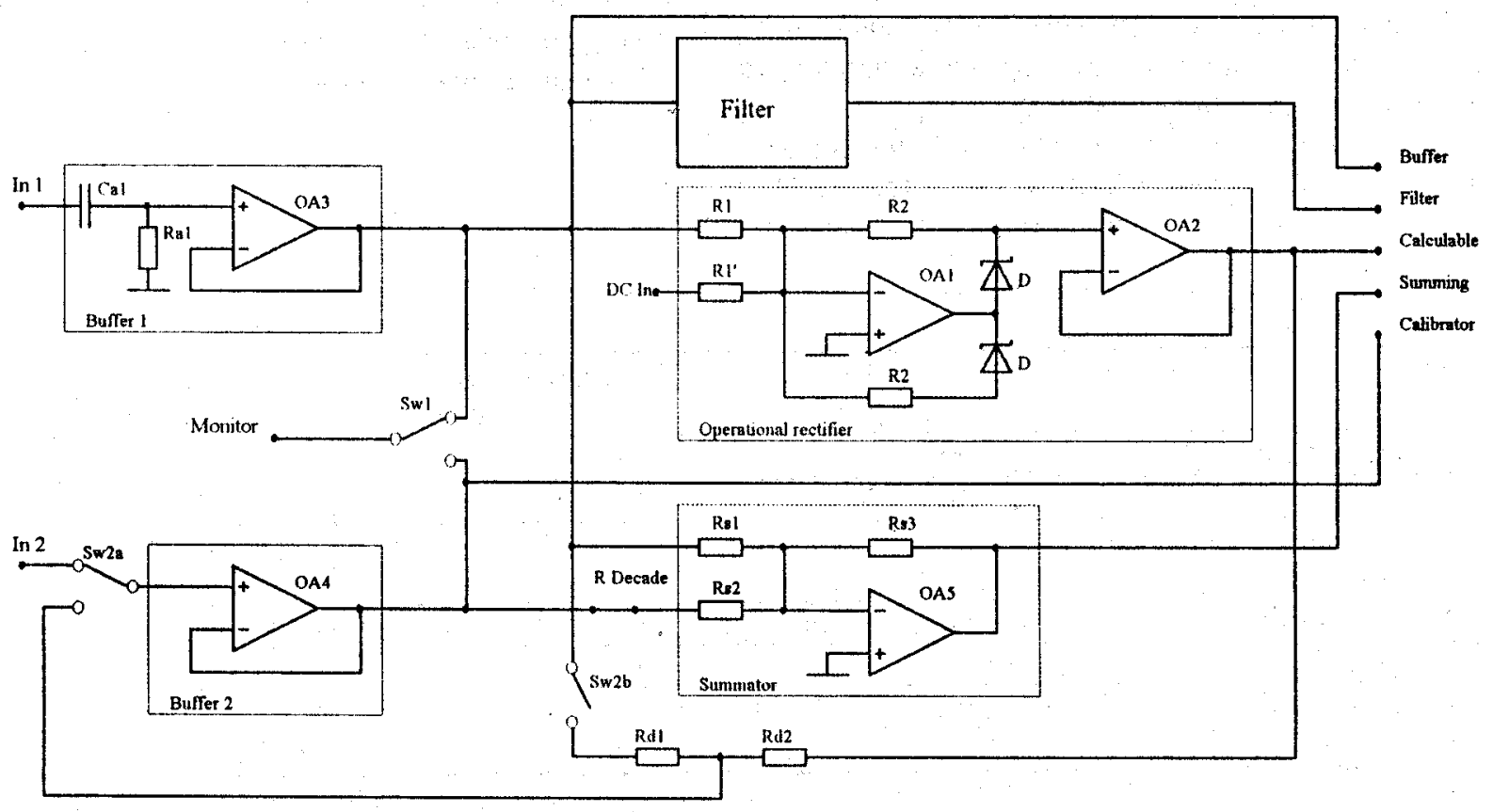

Fig. 11. Multifunction THD standard principle block diagram 
with a precision-limiting level setting (smoothly adjustable THD value)

- A summator without an external $R$ decade

- A summator with an external $R$ decade (for small THD values)

- A calibrator (discrete THD values)

- A calibrator with an external R decade (smoothly adjustable THD value).

We have measured each important parameter of the realized functional sample. The results are presented in [6] and some of them for calculable standard in [4]:

\subsection{Specifications}

The multifunctional THD standard specifications are based on the theoretical analyses and experimental verifications. They are shown in a simplified form in Tab. 1.

\section{Conclusions}

For the calibration of total harmonic distortion meters we need a generator with a known distortion - the standard of a distorted signal. Signals with known THD can be made in different ways, the following principles are preferred for metrology: a calculable standard, a standard based on summing two harmonic signals and a standard based on summing pure harmonic signal and the same signal distorted in a non-lincar circuit.

Simple distorted signals with known harmonics can be made by summing up two harmonic signals. The standard, in which the distortion can be set by the ratio of two resistors values, can be made by summing a pure harmonic signal and the same signal distorted in a nonlinear circuit. A calculable standard of the distorted signal can be simply made with a one-side limiter which can be realized by the application of a pure harmonic signal to an operational rectifier with a DC shift.

Based on the above-mentioned THD standard principles, a multifunctional THD standard for the THD meter calibration has been realized. Six different principles and circuit connections have been developed enabling a verification of the achieved results not only by measurements and calibrations but also by mutual comparisons. The developed multifunctional THD standard is now used in the Czech Metrological Institute.

\section{Acknowledgement}

This work has been supported by the grant No. 102/94/0351 from the Grant Agency of the Czech Republic.

\section{References}

[1] Metzler, B.: Audio measurement handbook. Audio Precision, Inc. 1993.

[2] Horský, P.: Calculable standard of non-linear distortion (in Czech). in. Proc. Radioelektronika '95, TU Brno, 1995, pp. 289. 292.

[3] Horský, J. - Horský, P.: Calibration of Multifunctional and Multirange Measuring Instruments Using Functional Blocks. Cal Lab - the international journal of metrology, March April 1997, pp. 33-36.

[4] Horský, P.: Multifunctional THD standard. in. Proc. Radiolektoronika '97, TU Bratislava, 1997, pp. 134-137.

[5] Calibration: Philosophy in practice. Second edition, Fluke Corp. 1994.

[6] Horský, P. - Horský, J.: THD standard. Czech Metrological Institute, Brno, Tech. rep. on Grant project work, Dec. 1996.

\section{About Author...}

Pavel HORSKÝ was born in Brno, in 1970. He received the M.S. degree in Electrical engineering from the Technical University of Brno in 1994. His research interests include circuit theory and applications, computeraided analysis and metrology

Tab. 1. Multifunctional THD standard specifications

\begin{tabular}{|l|l|l|l|l|}
\hline \multicolumn{1}{|c|}{ Standard } & \multicolumn{1}{|c|}{$\begin{array}{c}\text { THD } \\
{[\%]}\end{array}$} & $\begin{array}{c}\text { Relative THD error } \\
\text { limit [\%] }\end{array}$ & \multicolumn{1}{|c|}{$\begin{array}{c}\text { Relative THD error } \\
\text { typical [\%] }\end{array}$} \\
\hline 1 & Pure harm. Signal & $<10 \mathrm{ppm}$ & - & THD $\approx \mathrm{ppm}$ \\
\hline 2 & Summing & $0.1 \div 100$ & $0.1 \div 2$ & $0.007 \div 0.02$ \\
\hline 3 & $\begin{array}{l}\text { Summing with } \\
\text { external R }\end{array}$ & $\begin{array}{l}0.01 \\
0.001\end{array}$ & $\begin{array}{l}5 \\
200\end{array}$ & Depends on $\mathrm{R}$ \\
\hline 4 & Calculable & $7 \div 74($ discrete values) & $0.2 \div 10$ & $0.05 \div 2$ \\
\hline 5 & Limiter & $1 \div 100$ & $1 \div 10$ & $0.2 \div 3$ \\
\hline 6 & Calibrator & $0.03,0.1,0.3,1,3,10,39.9$ & $2.5 \div 10$ & $0.1 \div 2.5$ \\
\hline 7 & Calibrator wilh exl. R & $0.03 \div 100$ & $2.5 \div 10$ & Depends on $\mathrm{R}$ \\
\hline
\end{tabular}

For frequency range $10 \mathrm{~Hz} \div 100 \mathrm{kHz}\left(20 \mathrm{~Hz} \div 20 \mathrm{kHz}\right.$ for typical spec.), voltage $0.1 \div 5 \mathrm{~V}_{\mathrm{RMS}}$. 